$1-1-1951$

\title{
Effect of black walnut trees and their products on other vegetation
}

Maurice Brooks

Follow this and additional works at: https://researchrepository.wvu.edu/ wv_agricultural_and_forestry_experiment_station_bulletins

\section{Digital Commons Citation}

Brooks, Maurice, "Effect of black walnut trees and their products on other vegetation" (1951). West Virginia Agricultural and Forestry Experiment Station Bulletins. 347.

https://researchrepository.wvu.edu/wv_agricultural_and_forestry_experiment_station_bulletins/349 @ WVU. It has been accepted for inclusion in West Virginia Agricultural and Forestry Experiment Station Bulletins by an authorized administrator of The Research Repository @ WVU. For more information, please contact ian.harmon@mail.wvu.edu. 


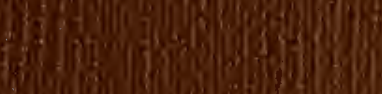

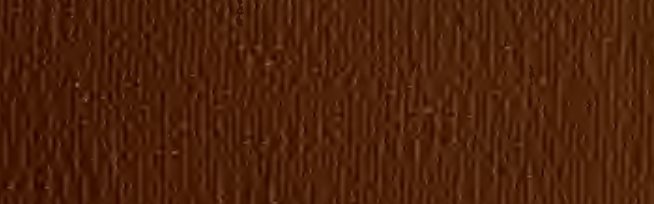

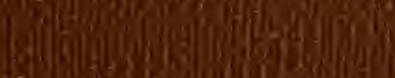

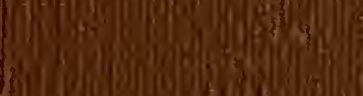
(f)

(1)

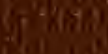

s.y.

Tholy

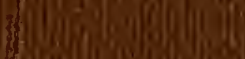

(1)

ong 
Digitized by the Internet Archive in 2010 with funding from

Lyrasis Members and Sloan Foundation 


\section{EFFECT OF BLACK WALNUT TREES}

\section{and their products on other vegetation}

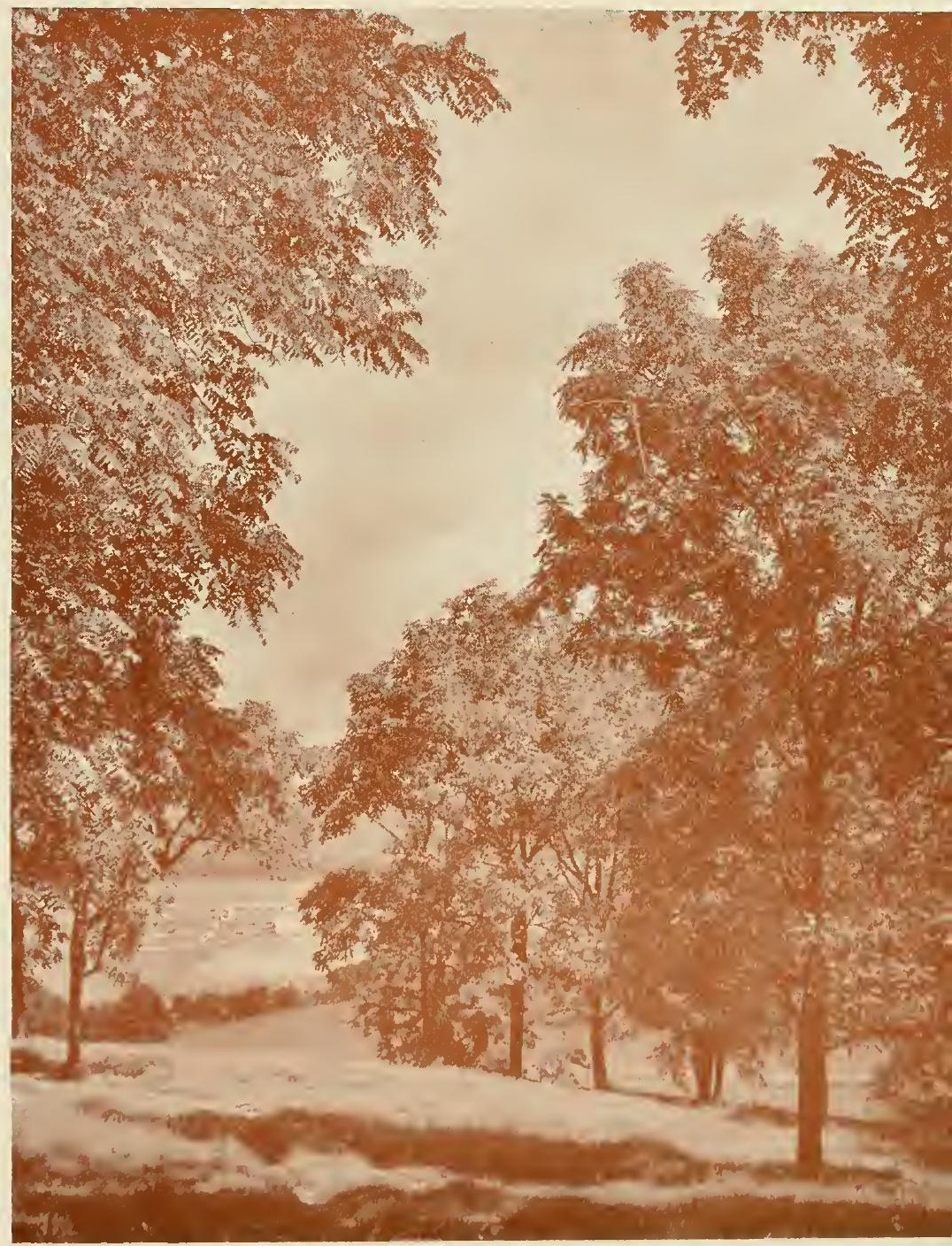

$\frac{B}{2}$

i

c 


\begin{abstract}
NAURICE G. BROOKS, author oi Ejject of Black ITalnut Trees and Their Products on Other legetation, is a Forester at the West Virginia University Agricultural Experiment Station and Professor of Wildlife Management in the College of Agriculture, Forestry. and Home Economics.
\end{abstract}

WEST VIRGINA UNIVERSITI

Agricultlral Experinient Station

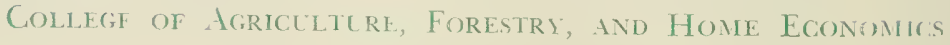

H. R. VARney; Director

MORGANTOWN 


\section{Contents}

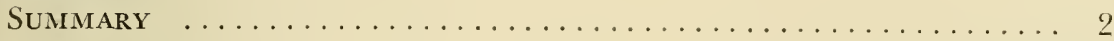

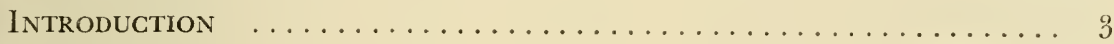

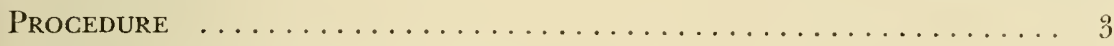

Range, Site, and Growth Habits $\ldots \ldots \ldots \ldots \ldots \ldots \ldots \ldots \ldots$. 5

Relationship to Other Plants $\ldots \ldots \ldots \ldots \ldots \ldots \ldots \ldots \ldots \ldots$

Occurrence of Plants Under Black Walnut ............ 10

Antagonisms With Other Plants ................. 10

Review of Literature .................... 16

Black Walnut and Other Forest Trees .............. 17

Black Walnut, Shrubs, and Vines $\ldots \ldots \ldots \ldots \ldots \ldots \ldots \ldots$

Black Walnut and Grasses .................... 18

Black Walnut and Grain Crops ................. 19

Black Walnut, Other Herbaceous Plants .............. 19

Black Walnut and Apple ..................... 20

Black Walnut, Other Fruit Trees $\ldots \ldots \ldots \ldots \ldots \ldots \ldots \ldots \ldots$

Black Walnut and Tomatoes and Potatoes ............. 21

Black Walnut and Alfalfa ...................... 23

Black Walnut and Blackberries ..................... 24

Black Walnut and Ericaceous Plants ................. 24

Other Walnut Species and Possible Antagonisms .......... 25

Nature of Toxic Agent in Black Walnut ................ 26;

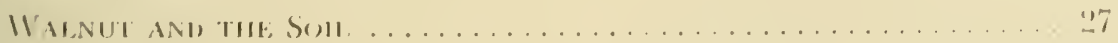

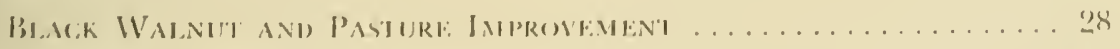

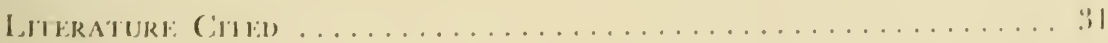




\section{SUMMARY}

THIS bulletin presents the results of field studies of three hundred black walnut trees (Juglans nigra L.) in relationship with their surrounding vegetation. Trees studied were in West Virginia, Virginia, Maryland, Ohio, and Michigan.

Data were taken on woody species, shrubs and vines, and herbaceous species occurring within circles of sixty-foot radius (approximately one-fourth acre) surrounding each tree.

Tables are given showing the frequency of occurrence, both within and without the root spread of walnut trees, of sixty tree species, thirtyfive shrubs and vines, and one hundred twenty-three herbaceous plants. Observations are given on apparent antagonisms between black walnut trees and apple, potatoes, tomatoes, alfalfa, blackberry, heaths, and other plants.

From evidence collected in this study, and from supporting evidence in literature, it is concluded that black walnut trees often exert a detrimental effect on certain other plants growing within the walnut's root spread.

Evidence indicates that actual contact with walnut roots is necessary before associated plants are harmed by walnuts.

The beneficial effect of black walnut on pastures in encouraging the growth of Kentucky bluegrass (Poa pratensis L.) and other cultivated grasses has been given. 


\section{EFFECT OF BLACK WALNUT TREES and their products on other vegetation}

MAURICE G. BROOKS

\section{INTRODUCTION}

THIS bulletin presents the results of, and conclusions drawn from, a field study of the black walnut (Juglans nigra L.), with particular reference to the relationships between black walnut trees and other plants. It also includes notes and observations on relationships between black walnuts and the soil, and the effect of standing walnut trees on pastures.

The primary object of this study was to determine the effect of standing black walnut trees and their products on woody and herbaceous vegetation. Field work began in the summer of 1939, and has been continued during the summers since that time. Detailed observations have been made on three hundred black walnut trees, all at least twelve inches in diameter. These three hundred trees were located in fortytwo West Virginia counties (all except Boone, Brooke, Fayette, Lincoln, Logan, McDowell, Mercer, Mingo, Nicholas, Ohio, Tucker, Wayne, and Wyoming); in five Virginia counties (Alleghany, Bath, Frederick, Giles, and Highland); in three Ohio counties (Jefferson, Meigs, and Washing(on); in two Maryland counties (Alleghany and Garrett), and, for purposes of comparison, in Washtenaw County, Michigan.

\section{PROCEDURE}

NOTES were taken at the selected trees on height of trees, diameter, $N_{\text {cover of area, exposure, slope, nature and } \mathrm{pH} \text { of the soil, within }}$ and without the root spread of the trec, drainage, altitude, and whether or not pastured. Within a circle of sixty foot radius from the trunk of the tree (all area of approximately onc-quarter acre) the crown spread of the tree was plotted, and an attempt made to list by species 
all trees, shrubs and vines, and herbaceous species present in significant quantities. For greater accuracy, check areas of one meter diameter were laid out at the cardinal compass points both within and without the root spread of the trees. Detailed analyses were made of the vegetation within these plots.

One set of check plots was placed within the root spread of each tree, ten feet north, east, south, and west of the trunk. Another set of check plots was located at the cardinal points of the compass five feet within the periphery of the sixty-foot-radius circle. In practically every case the latter plots were outside the root spread of the tree.

A field data sheet was prepared to allow convenient recording of significant information. This sheet, one copy of which was used for each tree surveyed, is in the form of a four-page folder. On page 1 (Figure 1) general data were recorded. On page 2 the crown spread

\section{BLACK WALNUT VEGETATION SURVEY}

1. Date

3. Height

5. Cover of Area
a. grassy pasture
b. meadow
c. brushy
d. woods

7. Exposure
a. open
b. sheltered
c. north
d. east
e. south
f. west

9. $\mathrm{pH}$
a. $5^{\prime}$ from trunk
b. $5^{\prime}$ outside spread

11. Pastured
a. light
b. heavy

2. Serial No.

4. D. b. h.

6. Slope
a. level
b. moderate
c. steep

8. Soil
a. litter
b. duff
c. clay loam
d. sand loam
e. gravelly

10. Drainage
a. good
b. poor

12. Altitude

1.3. Location
a. state
b. county
c. district
d. nearest P. O
e. farm

Figure 1. Field data sheet, page 1. Used in black walnut study. 
of the tree was plotted, and vegetation occurring both within and without the crown spread was indicated by key number as shown on page 3 in Figure 2. Page 4 of the folder was used for general remarks.

Most of the work was carried on in the Ohio Valley, but a considerable number of trees in the Potomac and James river basins also was investigated. Michigan trees examined were entirely outside the area covered by the main study. In the Appalachian region two physiographic provinces, the Valley and Ridge, and the Allegheny Plateau, were represented. An effort was made to select trees showing the widest possible range in altitude, and growing in the greatest possible variety of sites. Field studies were made at different times during the growing season so that account could be taken of varying floral aspects.

\section{RANGE, SITE, AND GROWTH HABITS}

Range. Black walnut occurs naturally throughout a wide area of eastern North America. It is found from northern New England, through southern Canada, and west to South Dakota; thence southward to central Texas; eastward to central Georgia; thence northward, reaching the Atlantic coast in South Carolina. It does not occur at high elevations in the Appalachian mountains region, but elsewhere it is well distributed throughout its botanical range.

The primary commercial range of the species as shown by Baker (2) also is extensive. Its center is in the Ohio Valley, but it extends to Arkansas.

West Virginia lies within the botanical range of the black walnut. Although the tree does not occur at high elevations, its range in southern West Virginia coincides roughly with the 3,000-foot contour, and in northern West Virginia with the 2,500-foot contour. According to Baker (2) only the western portion of the State falls within the primary commercial range of the species, although there are valleys from Mincral County, southward through Pendicton County, to Greenbrier and Monroe counties where black walnut is of commercial importance.

Site. Black walnut is a characteristic species of the "cove-type" forcst. It reaches its best growtl in the moist, rich soils that are characteristic of such arcas. Here it associates with tulip poplar (Liriodendron tulipifera L.), American chestnut (Castanea denlala [Marsh.] lorkh.), white ash (Fraximus americana L..), Basswood (Tilia spp.), black cherry (Prunus serotina Ehrh.), and a number of species of oak (Quercus spp.) and hickory (Carya spp.). 


\section{DETAIL MAP OF AREA}

\section{Approximate Countour of Tree Spread in Blue Pencil}

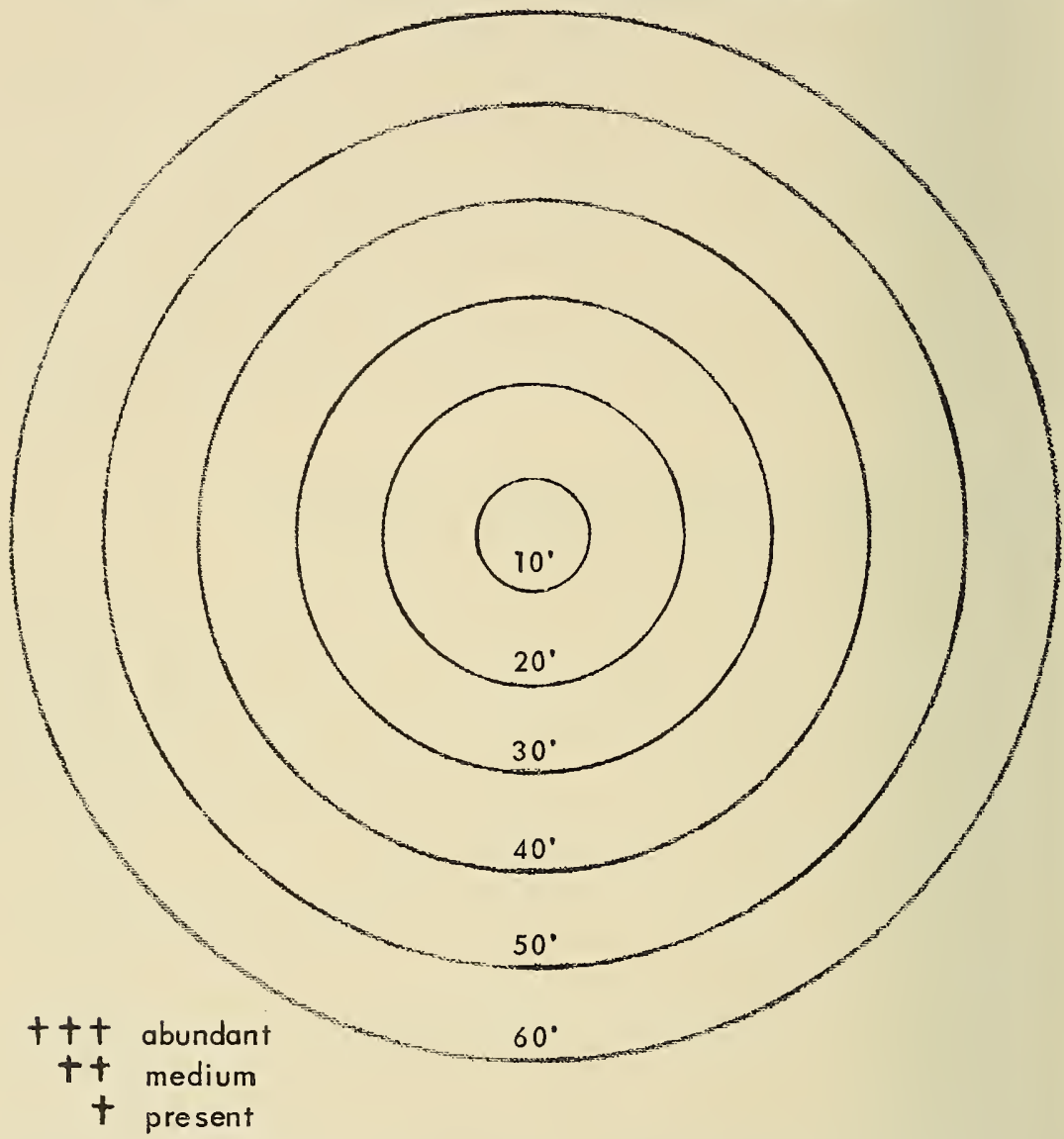

Figure 2. Page 2 of Field Data Sheet used in black walnut study. Page 3 of data sheet is shown on opposite page. Approximate crown spread of tree is plotted and indicated in blue pencil on page 2 of data sheet. Location of various tree species is indicated by number from key on opposite page. These numbers are made with black pencil and enclosed by broken circle. Shrub numbers also are marked with black pencil on data sheet, but enclosed in small rectangle. Herb species numbers are indicated in red pencil and enclosed by broken circle. Where a species is present, an $\uparrow$ is marked beside its number; $\dagger \dagger$ denotes the species to be fairly well established; and $+\dagger \dagger$ indicates the species is abundant. 


\section{KEY}

Black Pencil

Red Pencil

Tree

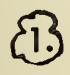

Shrub

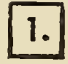

Herbaceous

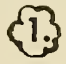

\section{TREES}

1. P. Pine

2. S. Pine

3. R. Cedar

4. B. Willow

5. L-t. Aspen

6. W. Walnut

7. S-b. Hickory

8. Mockernut

9. Pignut

10. Bitternut

11. B. Birch

12. Beech

13. Chestnut

14. W. Oak

15. C. Oak

16. R. Oak

17. Scar. Oak

18. B. Oak

19. S. EIm

20. A. Elm

21. Cucumber

22. Tulip

23. Pawpaw

24. Sassafras

25. W. Hazel

26. Sycamore

27. Crab Apple

28. Service

29. Crataegus

30. B. Cherry

31. H. Locust

32. Red Bud

33. B. Locust

34. S. H. Sumac

\section{TREES}

35. Sug. Maple

36. SIv. Maple

37. Red Maple

38. Box Elder

39. Buckeye

40. Basswood

41. F. Dogwood

42. B. Gum

43. Sourwood

44. Persimmon

45. W. Ash

46. B. Haw

47.

48.

49.

59.

SHRUBS

1. D. Sumac

2. S. Sumac

3. Hazelnut

4. Pipevine

5. Barberry

6. Spicebush

7. Hydrangea

8. Gooseberry

9. Poison Ivy

10. Bittersweet

11. W. Grape

12. Sk. Dogwood

13. Viburnum

14. Elder

15. Blackberry

\section{SHRUBS}

16. B. Raspberry

17. W. Rose

18.

19.

20.

21.

\section{HERBS}

1. C. Bluegrass

2. K. Bluegrass

3. Thistle

4. Solidago

5. Aster

6. Senecio

7. Ironweed

8. Erigeron

9. Eupatorium

10. C. Fern

11. Ebony Sp.

12. Botrychium

13.

14.

15.

16.

ADJACENT VEGETATION

Trees (Use Nos. Above)

Shrubs (Use Nos. Above)

Herbs

1. Broomsedge

2. Danthonia

3.

4.

5.

6. 
Within the region covered by this study, however, the black walnut is not restricted to such areas. West Virginia is not too far from the center of the species' botanical range. The State offers some growing conditions that are near optimum for the species. Walnut often grows well up toward the tops of the hills, on steep slopes, and in comparatively dry sites. It is a common associate of black locust (Robinia pseudoacacia L.) in hilly pastures.

Black walnut is highly intolerant. It is not commonly found in dense woods. Near the borders of woods it becomes much more abundant, and because it often is left standing when land is cleared, it is frequently found in pure scattered stands in pastures. It also is characteristic of fencerows, since squirrels regularly bury the nuts in such locations, and since the seedlings are likely to receive greater protection in such situations.

Many soils seem suitable for walnut growth, although growth is decidedly slower on poor, thin, dry soils. The tree flourishes in limestone areas as well as in soils of moderate acidity.

Growth Habits. Black walnut is a medium to large tree, averaging in our region 70-80 feet in height, and 2-3 feet in diameter. Trees 150 feet high and six feet in diameter have been reported. The tree is normally very high crowned. The crown is narrow under forest conditions, but much more spreading in trees located in open areas. This high crown results in trunks that are often free of branches for thirty feet or more.

Seedlings develop a deep taproot. As the tree grows, a heavy lateral root system also is produced. The lateral roots are usually well under the ground, an important feature in relationship to herbaceous cover under the trees, since competition for moisture is thereby reduced.

There is considerable variation among individual trees in the time of blooming. This is significant in the Appalachian region where late frosts occur. The nuts from different trees also vary widely in size, thickness of shell, shape, flavor, and viability.

\section{RELATIONSHIP TO OTHER PLANTS}

RLACK walnut is remarkable in its relationship with other plants. Vegetation under walnut trees is likely to differ sharply from that outside the crown and root spread of the trees.

For example, on a farm in Upshur County there is a large walnut tree, about 70 feet high, 3 feet diameter breast high, and with a rather uniform crown spread of about 70 feet. The tree stands in a brushy 
pasture field, and outside its spread the soil is generally poor and thin, with the vegetative cover consisting largely of three species, broomsedge (Andropogon virginicus L.), poverty grass (Danthonia spicata [L.] Beauv.), and blackberry (Rubus spp.). The better pasture grasses, and even weeds useful to sheep, are scarce or absent.

Under the tree, however, extending inward from the outer edge of the root spread, there is a court of Kentucky bluegrass (Poa pratensis L.), with some timothy (Phleum pratense L.), and redtop (Agrostis alba L.). Near the trunk is a compact stand of black raspberry (Rubus occidentalis L.). Within twenty feet of the trunk, broomsedge, poverty grass, and blackberry are absent.

Other plants present include fleabane (Erigeron), three species of fern (Botrychium obliquum, Muhl., Polystichum acrostichoides [Michx.] Schott., and Asplenium platyneuron [L.] Oakes.), and various asters, goldenrods, mints, violets, and a few clovers. All of these are relatively abundant within the spread of the tree, but scarce or absent outside it.

This particular tree is on an open slope, not in a cove of richer soil. There is no evidence that it receives more moisture than the surrounding area. Outside the spread of the tree, but within the 60 -foot circle, soil tests show a pH of 5.1. Within the root spread, however, the $\mathrm{pH}$ is 5.8. The soil has not received either lime or fertilizer during the last fifty years. The tree court and its surroundings received exactly the same treatment; both were pastured fairly heavily until about fifteen years ago, but since then there has been no grazing.

The line of demarcation between the broomsedge-poverty grassblackberry stand outside and the grass court under the tree is sharp and definite. Surrounding the tree is an island of high-class vegetation in an expanse of worthless grasses, weeds, and briers. Other than the tree, there are no other factors of site that would account for these differences.

This is not an isolated example. On limestone soils, however, the line of demarcation between tree court and surroundings often is not so sharp. On acid soils the picture holds; no matter how poor the surroundings may be. Within the spread of the tree there are better grasses and a highly characteristic group of hcrbs and woody plants. Prominent among these are black raspberry, wild grapes (Vitis spp.), Virginia creeper (Psedera quinquefolia [L.] Greenc.), clovers (Trifolium spp.), fleabane, thistle (Cirsium spp.), violets, ferns, asters, goldenrods, mints, and ironweed (Vernonia glauca [L.] Willd.) .

The most prominent grass under black wahunt trees is Kentucky bluegrass. Other cultivated grasses of importance are redtop, timothy, 
tall oat grass (Arrhenatherum elatius [L.] Beauv.), orchard grass (Dactylis glomerata L.), and meadow fescue (Festuca elatior L.). Canada bluegrass (Poa compressa L.) is rather uncommon under black walnut, occurring only in drier and more open situations. Smith (15) has pointed out the virtual absence of poverty grass under black walnut, although it grows freely under many other trees in open pastures. $\mathrm{He}$ also pointed out the absence of broomsedge under walnuts. Wild grasses of rather frequent occurrence under walnut include broadleaved panic grass (Panicum clandestinum L.), nimblewill (Muhlenbergia schreberi J. F. Gmel.), velvet grass (Holcus lanatus L.), and purpletop (Triodia fava R. Br.). These and other wild grasses are usually much less prominent than are the more desirable species.

\section{Occurrence of Plants Under Black Walnut}

As previously noted, black walnut trees have been surveyed intensively as to the vegetation that occurs within their root spread. Table 1 lists all the tree species found growing under walnut. Table 2 lists all the shrubs and vines, including semi-woody species such as blackberry and black raspberry. Table 3 lists the principal herbaceous species found under ten or more trees. In some cases identification of species was difficult or impossible owing to sterile condition of the plants, natural difficulty of separation within the group, etc. In such cases the plants are referred to the genera only. In the three tables the numbers and per cent of occurrence are given for each plant.

Table 4 compares the frequency of occurrence of certain representative plants within and outside the crown spread of the trees, but within the sixty-foot circle around each tree. These frequencies were determined through comparisons of the small check-plots already described or through observation in the case of larger woody plants.

In view of the reported antagonisms between black walnut and other plants in a wide variety of families, a special effort was made to determine what plants do occur in association with walnut. This accounts for the detail as seen in Tables 1-4.

\section{Antagonisms With Other Plants}

The question of antagonisms between black walnut and other plant species is much disputed. Some observers consider the apparent injury done to many plants when they are associated with black walnut to be the natural results of shade and competition for water. Most investigators of black walnut relations, however, are convinced that antagonisms do exist, and that these have a chemical and physiological basis 
Table 1. Forest Tree Species Growing within Root Spread of 300 Black Walnut Trees

\begin{tabular}{|c|c|c|}
\hline SPECIES & OCCURRENCES & $\begin{array}{c}\text { PER CENT OF } \\
\text { OCCURRENCE }\end{array}$ \\
\hline White Pine (Pinus Strobus) & 4 & 1.3 \\
\hline Pitch Pine (P. rigida) & 1 & 0.3 \\
\hline Serub Pine (P. virginiana) & 12 & 4.0 \\
\hline Red Pine (P. resinosa) (In plantations) & 2 & 0.7 \\
\hline Norway Spruce (Picea abies) (In plantations) & 3 & 1.0 \\
\hline Hemlock (Tsuga canadensis) & 2 & 0.7 \\
\hline Arbor Vitae (Thuja occidentalis) & 1 & 0.3 \\
\hline $\mathrm{R} \subseteq \mathrm{d}$ Cedar (Juniperus virginiana) & 17 & 5.7 \\
\hline Black Willow (Salix nigra) ............ & 8 & 2.7 \\
\hline White Walnut (Juglans cinerea) & 7 & 2.3 \\
\hline Hickory (Carya sp.) & 21 & 7.0 \\
\hline American Hornbeam (Carpinus caroliniana) & 6 & 2.0 \\
\hline Black Birch (Betula lenta) & 13 & 4.3 \\
\hline Yellow Birch (B. lutea) & 2 & 0.7 \\
\hline River Birch (B. nigra) & 3 & 1.0 \\
\hline Beech (Fagus grandifolia) ...... & 34 & 11.3 \\
\hline Cbestnut (Castanea dentata) & 2 & 0.7 \\
\hline White Oak (Quercus alba) ....... & 27 & 9.0 \\
\hline Post Oak (Q. stellata) ................. & 1 & 0.3 \\
\hline Red Oak (Q. borealis) & 11 & 3.7 \\
\hline Scarlet Oak (Q. coccinea) & 6 & 2.0 \\
\hline Elack Oak (Q. velutina) & 14 & 4.7 \\
\hline Sbingle Oak (Q. imbricaria) .............. & 1 & 0.3 \\
\hline Slippery Elm (Ulmus fulva) ............... & 5 & 1.7 \\
\hline American Elm (U. americana) ........ & 41 & 13.7 \\
\hline Hackberry (Celtis occidentalis) .............. & 4 & 1.3 \\
\hline Red Mulberry (Morus rubra) & 3 & 1.0 \\
\hline Cucumber Tree (Nragnolia acuminata) & 5 & 1.7 \\
\hline Tulip Tree (Liriodendron tulipifera) ..... & 26 & 8.7 \\
\hline Papaw (Asimina triloba) & 12 & 4.0 \\
\hline Sassafrass (Sassafrass variifolium) ......... & 20 & 6.7 \\
\hline Witch-bazel (Hamamelis virginiana) .. & 6 & 2.0 \\
\hline Sweet Gum (Liquidambar styraciflua) .. & 2 & 0.7 \\
\hline Sycamore (Platanus occidentalis) ........... & 27 & 9.0 \\
\hline American Crab (Pyrus coronaria) & 13 & 4.3 \\
\hline Service Berry (Amelanchier canadensis) & 7 & 2.8 \\
\hline Hawthorn (Crataegus spp.) & 46 & 15.3 \\
\hline Black Cherry (Prunus serotina) & 22 & 7.3 \\
\hline Fire Cherry (P. pennsylvanica) ....... & 1 & 0.3 \\
\hline Wild Plum (Prunus americaua) ....... & 8 & 2.7 \\
\hline IJoney Locust (Gleditsia triacantbos) & 10 & 3.3 \\
\hline 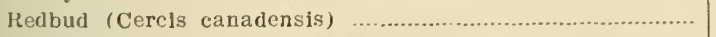 & 19 & 6.3 \\
\hline Locust (Robinia pseudoacacia) ................. & 32 & 10.3 \\
\hline Tree of Heaven (Allanthus glandulosa) & 15 & 5.0 \\
\hline Staghorn Sumac (Rhus hirta) ................. & 20 & 6.7 \\
\hline Anierican Holly (Ilex opaca) .............. & 2 & 0.7 \\
\hline Sugar Waple (Acer saccharum) ......... & 19 & 6.3 \\
\hline 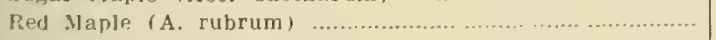 & 23 & 7.7 \\
\hline 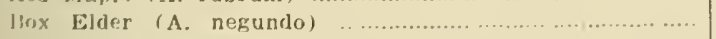 & 4 & 1.3 \\
\hline 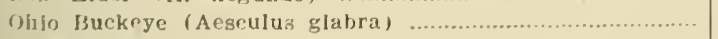 & 12 & -1.11 \\
\hline 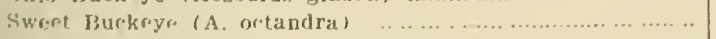 & 2 & 11.7 \\
\hline 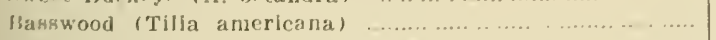 & 4 & 1,3 \\
\hline White baskwood ( $\mathrm{T}$. heterophylla) ........... & 1 & $11, . ;$ \\
\hline l'Iowrering Dogwood (Cornug florida) ................. - & 19 & $1: . .3$ \\
\hline 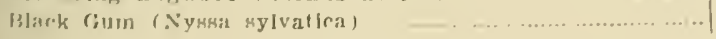 & 11 & $\therefore .7$ \\
\hline
\end{tabular}




\begin{tabular}{|c|c|c|}
\hline SPECIES & OCCURRENCES & $\begin{array}{l}\text { PER CENT OF } \\
\text { OCCURRENCE }\end{array}$ \\
\hline 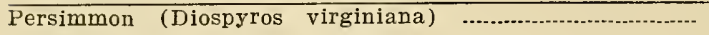 & 3 & 1.0 \\
\hline Opossum Wood (Halesia carolina) & 2 & 0.7 \\
\hline White Ash (Fraxinus americana) & 8 & 2.7 \\
\hline Black Ash (F. nigra) & 1 & 0.3 \\
\hline Black Haw (Viburnum prunifolium) & 14 & 4.7 \\
\hline
\end{tabular}

Tree species are listed in order of their classification in Gray's New Manual of Botany, not in order of their abundance. The list includes all size classes from seedlings to large trees.

\section{Table 2. Shrubs and Woody Vines Growing within Root Spread of 300 Black Walnut Trees}

\begin{tabular}{|c|c|c|}
\hline SpeCIES & OCCURRENCES & $\begin{array}{c}\text { Per Cent of } \\
\text { OCCURRence }\end{array}$ \\
\hline Hazelnut (Corylus Americana) ..... & 14 & 4.7 \\
\hline Smooth Alder (Alnus rugosa) & 2 & 0.7 \\
\hline Pipe Vine (Aristolochia macrophylla) ...... & 3 & 1.0 \\
\hline Virgin's Bower (Clematis virginiana) .... & 11 & 3.7 \\
\hline American Barberry (Berberis canadensis) .... & 16 & 5.3 \\
\hline Spice Bush (Benzoin aestivale) & 63 & 21.0 \\
\hline Wild Hydrangea (Hydrangea arborescens) & 54 & 18.0 \\
\hline Currant (Ribes spp.) & 31 & 10.3 \\
\hline Nine-bark (Physocarpus opulifolius) & 1 & 0.3 \\
\hline Black Raspberry (Rubus occidentalis) ........... & 184 & 61.3 \\
\hline Purple Flowering Raspberry ( $R$. odoratus) ..... & 3 & 1.0 \\
\hline Blackberry (Rubus spp.) & 2 & 0.7 \\
\hline Wild Rose (Rosa spp.) & 89 & 29.7 \\
\hline Choke Cherry (Prunus virginiana) & 5 & 1.7 \\
\hline Prickly Ash (Zanthoxylum americanum) .... & 2 & 0.7 \\
\hline 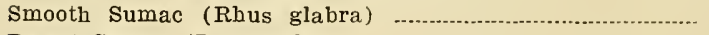 & 37 & 12.3 \\
\hline Dwarf Sumac (R. copallina) & 46 & 15.3 \\
\hline Poison Ivy (R. toxicodendron) & 115 & 38.3 \\
\hline 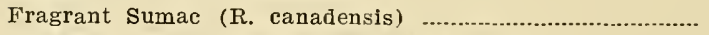 & 3 & 1.0 \\
\hline Strawberry Bush (Evonymus americanus) .......... & 5 & 1.7 \\
\hline Climbing Bitter-sweet (Celastrus scandens) ...... & 25 & 8.3 \\
\hline Bladder Nut (Staphylea trifolia) & 2 & 0.7 \\
\hline New Jersey Tea (Ceanothus americanus) & 2 & 0.7 \\
\hline Virginia Creeper (Psedera quinquefolia) ............... & 123 & 41.0 \\
\hline Wild Grape (Vitis spp.) & 103 & 34.3 \\
\hline Shrubby St. John's-Wort (Hypericum prolificum). & 14 & 4.7 \\
\hline Hercules' Club (Aralia spinosa) & 3 & 1.0 \\
\hline 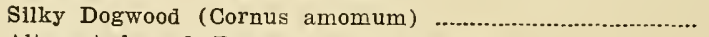 & 7 & 2.3 \\
\hline Alternate-leaved Dogwood (C. alternifolia) ........ & 13 & 4.3 \\
\hline Fringe-tree (Chionanthus virginica) & 1 & 0.3 \\
\hline American Fly Honeysuckle (Lonicera canadensis) ........ & 3 & 1.0 \\
\hline Maple-leaved Viburnum (Viburnum acerifolium) ............. & 21 & 7.0 \\
\hline Arrow-wood (Vlburnum dentatum) & 7 & 2.3 \\
\hline 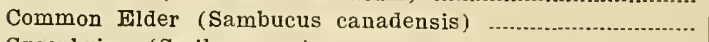 & 46 & 15.3 \\
\hline Greenbrier (Smilax spp.) & 63 & 21.0 \\
\hline
\end{tabular}
7 th ed.

Species are listed in order of their classification in Gray's New Manual of Botany, 

of 300 Black Walnut Trees

\begin{tabular}{|c|c|c|}
\hline SPECIES & OCCURRENCES & $\begin{array}{l}\text { Per Cent of } \\
\text { OCCURRENCE }\end{array}$ \\
\hline Ehony Spleenwort (Asplenium platyneuron) ... & 73 & 24.3 \\
\hline Silvery Spleenwort (Athryium thelypteroides) & 23 & 7.7 \\
\hline Lowland Lady Fern (Athryium asplenioides) .................... & 36 & 12.0 \\
\hline Christmas Fern (Polystichum acrostichoides) ........ & 91 & 30.3 \\
\hline Marginal Shield Fern (Dryopteris marginalis) & 41 & 13.7 \\
\hline 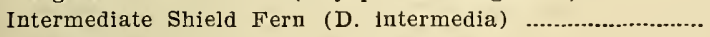 & 54 & 18.0 \\
\hline 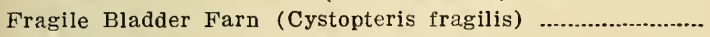 & 11 & 3.7 \\
\hline Obtuse Woodsia (Woodsia obtusa) & 18 & 6.0 \\
\hline Hay-scented Fern (Dennstaedtia punctilobula) ...... & 24 & 8.0 \\
\hline Sensitive Fern (Onoclea sensibilis) & 12 & 4.0 \\
\hline 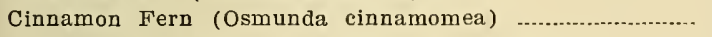 & 11 & 3.7 \\
\hline Dissected Grape Fern (Botrychium dissectum) ....... & 31 & 10.3 \\
\hline Common Grape Fern (B. dissectum var. obliquum) & 78 & 26.0 \\
\hline Common Horsetail (Equisetum arvense) & 12 & 4.0 \\
\hline $\begin{array}{l}\text { Ground Pine (Lycopodium complanatum var. } \\
\text { flabelliforme) }\end{array}$ & 19 & 6.3 \\
\hline Broomsedge (Andropogon virginicus) & 13 & 4.3 \\
\hline Broad-leaved Panic Grass (Panicum clandestinum) ........ & 38 & 12.7 \\
\hline Green Foxtail Grass (Setaria viridis) & 24 & 8.0 \\
\hline 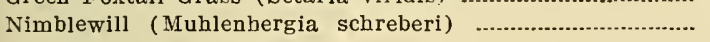 & 93 & 31.0 \\
\hline Timothy (Phleum pratense) & 69 & 23.0 \\
\hline Redtop (Agrostis alba) & 88 & 29.3 \\
\hline Hair Grass (Agrostic hyemalis) & 31 & 10.3 \\
\hline 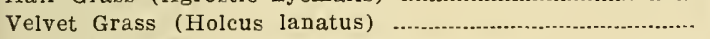 & 60 & 20.0 \\
\hline Tall Oat Grass (Arrhenatherum elatius). & 54 & 18.0 \\
\hline Poverty Grass (Danthonia spicata) ............ & 24 & 8.0 \\
\hline 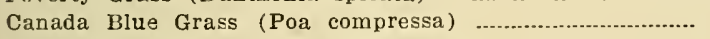 & 13 & 4.3 \\
\hline 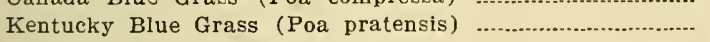 & 215 & 71.7 \\
\hline 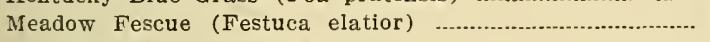 & 89 & 29.7 \\
\hline Brome Grass (Bromus spp.) & 51 & 17.0 \\
\hline 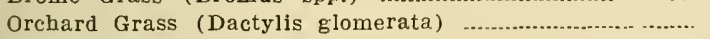 & 80 & 26.7 \\
\hline Sedges (Carex spp.) & 55 & 18.3 \\
\hline Jack-in-the-Pulpit (Arisaema triphyllum) & 24 & 8.0 \\
\hline Virginia Day-flower (Commelina communis) ........ & 15 & 5.0 \\
\hline 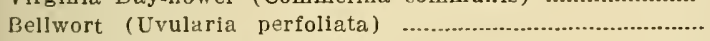 & 19 & 6.3 \\
\hline 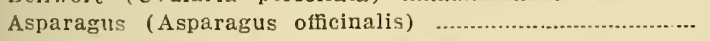 & 12 & 4.0 \\
\hline Ealse Spikenard (Smilacina racemosa) ...... & 15 & 5.0 \\
\hline Wild Yam (Dioscorea villosa) & 21 & 7.0 \\
\hline 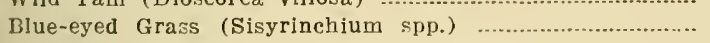 & 44 & 14.7 \\
\hline Ragged Fringed Orchid (IIabenaria lacera) ... & 10 & 3.3 \\
\hline Slender Ladies Tresses (Spiranthes gracilis) ...... & 41 & 13.7 \\
\hline 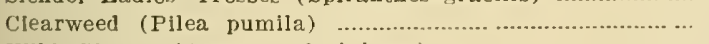 & 33 & 11.0 \\
\hline Wlld Glnger (Asarum virginicum) & 16 & 5.3 \\
\hline Dock (Rumex spp.) & 25 & 8.3 \\
\hline Mouse-ear Chickweed (Cerastlum vulgatum) ............ & 19 & 6.3 \\
\hline 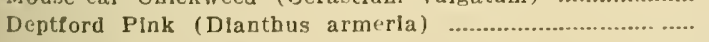 & 60 & 20.0 \\
\hline 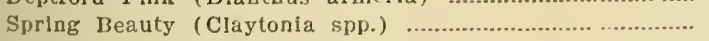 & 22 & 7.3 \\
\hline Crowloot (Ranunculus spp.) & 26 & 8.7 \\
\hline 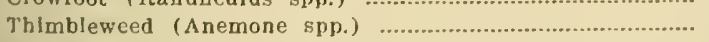 & 16 & 5.3 \\
\hline Moonsced (Menispermum canaderise) ... & 41 & 13.7 \\
\hline 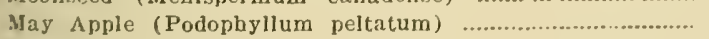 & 13 & 4.3 \\
\hline Mustard (Brassica spp.) & 23 & 7.7 \\
\hline 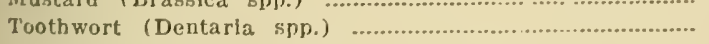 & 17 & 5.7 \\
\hline Stoncerop (Sedum ternatum) ..... & 26 & 8.7 \\
\hline
\end{tabular}




\begin{tabular}{|c|c|c|}
\hline SPECIES & OCCURRENCES & $\begin{array}{l}\text { Per Cent of } \\
\text { OCCURRence }\end{array}$ \\
\hline Alum Root (Heuchera spp.) & 10 & 3.3 \\
\hline Common Cinquefoil (Potentilla canadensis) ... & 77 & 25.7 \\
\hline Avens (Geum spp.) & 81 & 27.0 \\
\hline Wild Senna (Cassia marilandica) .......... & 15 & 5.0 \\
\hline 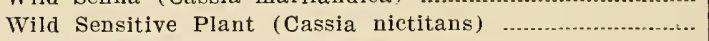 & 24 & 8.0 \\
\hline Red Clover (Trifolium pratense) & 62 & 20.7 \\
\hline White Clover ( $\mathrm{T}$, repens) & 131 & 43.7 \\
\hline Alsike Clover ( $\mathrm{T}$. hybridum) & 38 & 12.7 \\
\hline Hop Clover (Trifolium spp.) & 28 & 9.3 \\
\hline Sweet Clover (Melilotus spp.) ...... & 42 & 14.0 \\
\hline Tick Trefoil (Desmodium spp.) & 31 & 10.3 \\
\hline 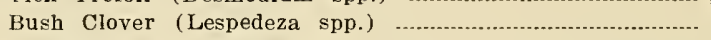 & 45 & 15.0 \\
\hline Wood Sorrel (Oxalis spp.) & 117 & 39.0 \\
\hline Wild Geranium (Geranium spp.) .............. & 33 & 11.0 \\
\hline Milkwort (Polygala sanguinea) .......... & 20 & 6.7 \\
\hline Jewelweed (Impatiens spp.) & 21 & 7.0 \\
\hline Common Mallow (Malva rotundifolia) ........ & 12 & 4.0 \\
\hline St. John's-wort (Hypericum spp.) & 37 & 12.3 \\
\hline Violet (Viola spp.) & 83 & 27.7 \\
\hline Clammy Cuphea (Cuphea petiola) ......... & 101 & 33.7 \\
\hline Evening Primrose (Oenothera spp.) ......... & 35 & 11.7 \\
\hline Wild Carrot (Daucus carota) & 112 & 37.3 \\
\hline Common Milkweed (Asclepias syriaca). & 100 & 33.3 \\
\hline Field Bindweed (Convolvulus arvensis) & 51 & 17.0 \\
\hline Dodder (Cuscuta spp.) & 43 & 14.3 \\
\hline Gill-over-the-ground (Nepeta hederacea) ........ & 62 & 20.7 \\
\hline 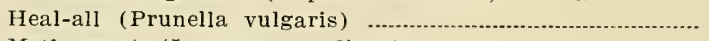 & 122 & 40.7 \\
\hline Motherwort (Leonurus cardiaca) ......... & 67 & 22.3 \\
\hline 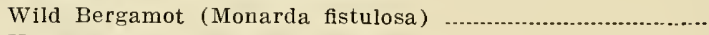 & 76 & 25.3 \\
\hline Horse Mint (Monarda punctata) & 49 & 16.3 \\
\hline Pennyroyal (Hedeoma pulegioides) ........ & 62 & 20.7 \\
\hline Mountain Mint (Pycnanthemum spp.) & 33 & 11.0 \\
\hline Peppermint (Mentha piperita) & 27 & 9.0 \\
\hline Rich-weed (Collinsonia canadensis) & 20 & 6.7 \\
\hline Horse Nettle (Solanum carolinense) ....... & 68 & 22.7 \\
\hline 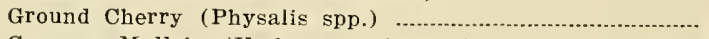 & 51 & 17.0 \\
\hline Common Mullein (Verbascum thapsus) ... & 71 & 23.7 \\
\hline 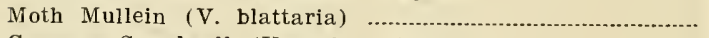 & 42 & 14.0 \\
\hline Common Speedwell (Veronlea officinalis) ............. & 46 & 15.3 \\
\hline Common Plantain (Plantago major) & 59 & 19.7 \\
\hline English Plantain (P. lanceolata) & 76 & 25.3 \\
\hline Bedstraw (Galium spp.) & 90 & 30.0 \\
\hline Tall Bellflower (Campanula americana) & 18 & 6.0 \\
\hline Great Lobelia (Lobelia siphilitica) & 39 & 13.0 \\
\hline Spiked Lobelia (L. spicata) & 21 & 7.0 \\
\hline Indian Tobacco (Lobelia inflata) ........ & 88 & 29.3 \\
\hline Ironweed (Vernonia spp.) & 122 & 40.7 \\
\hline Elephant's Foot (Elephantopus spp.) & 62 & 20.7 \\
\hline Joe-Pye Weed (Eupatorium purpureum) ............. & 51 & 17.0 \\
\hline Mist-flower (Eupatorium coelestinum) & 63 & 21.0 \\
\hline Goldenrod (Solidago spp.) & 133 & 44.3 \\
\hline Aster (Aster spp.) & 162 & 54.0 \\
\hline Philadelphia Fleabane (Erigeron philadelphicus) ............ & 30 & 10.0 \\
\hline Daisy Fleabane (E. annuus) & 115 & 38.3 \\
\hline Butter-weed (E. canadensis) & 03 & 21.0 \\
\hline Pussy's Toes (Antennaria spp.) & 58 & 19.3 \\
\hline Ragweed (Ambrosia spp.) & 124 & 41.3 \\
\hline
\end{tabular}




\begin{tabular}{|c|c|c|}
\hline SPECIES & OCCURRENCES & $\begin{array}{l}\text { Per Cent of } \\
\text { OCCURRENCE }\end{array}$ \\
\hline Brown-eyed Susan (Rudbeckia hirta) .. & 82 & 27.3 \\
\hline 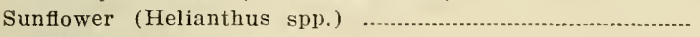 & 70 & 23.3 \\
\hline Beggar-ticks (Bidens spp.) & 102 & 34.0 \\
\hline Sneezeweed (Helenium spp.) & 54 & 18.0 \\
\hline Common Yarrow (Achillea millefolium) & 116 & 38.7 \\
\hline Ox-eye Daisy (Chrysanthemum leucanthemum) & 146 & 48.7 \\
\hline Golden Ragwort (Senecio aureus) & 127 & 42.3 \\
\hline Thistle (Cirsium spp.) & 105 & 35.0 \\
\hline Chicory (Cichorium intybus) .... & 44 & 14.7 \\
\hline Dandelion (Taraxacum spp.) & 113 & 37.7 \\
\hline Wild Lettuce (Lactuca spp.) & 92 & 30.7 \\
\hline Rattlesnake-root (Prenanthes spp.) & 71 & 23.7 \\
\hline Hawkweed (Hieracium spp.) & 85 & 28.3 \\
\hline
\end{tabular}

Species listed in order of their classification in Gray's New Manual of Botany, 8th ed.

Table 4. Comparison of Ogcurrence of Certain Plant Species within and Outside Crown-spread of 300 Black Walnut Trees

\begin{tabular}{|c|c|c|c|c|}
\hline \multirow[b]{2}{*}{ SPECIES } & \multicolumn{2}{|c|}{$\begin{array}{l}\text { WITHIN HORIZONTAL } \\
\text { PROJECTYON OF CROWN }\end{array}$} & \multicolumn{2}{|c|}{$\begin{array}{l}\text { OUTSIDE HORIZONTAL } \\
\text { PROJECTION OF CROWN }\end{array}$} \\
\hline & OCCURRENCES & $\begin{array}{c}\text { Per Cent } \\
\text { OCcurrence }\end{array}$ & OCCURRENCES & $\begin{array}{c}\text { PER CENT } \\
\text { OCCURRENCE }\end{array}$ \\
\hline Ebony Spleenwort & 73 & 24.3 & 21 & 7.0 \\
\hline Christmas Fern & 91 & 30.3 & 38 & 12.7 \\
\hline Common Grape Fern & 78 & 26.0 & 14 & 4.7 \\
\hline 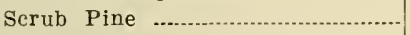 & 12 & 4.0 & 20 & 6.7 \\
\hline Broomsedge & 13 & 4.3 & 112 & 37.3 \\
\hline Broad-leaved Panic Grass ........... & 38 & 12.7 & 13 & 4.3 \\
\hline Nimblewill ................................... & 93 & 31.0 & 123 & 41.0 \\
\hline 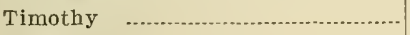 & 69 & 23.0 & 19 & 6.3 \\
\hline Redtop & 88 & 29.3 & 28 & 9.3 \\
\hline Velvet Grass & 60 & 20.0 & +1 & 13.7 \\
\hline Tall Oat Grass ........ & 54 & 18.0 & 21 & 7.0 \\
\hline Poverty Grass & 24 & 8.0 & 163 & 54.3 \\
\hline Canada Blue Grass .......... & 13 & 4.3 & 36 & 12.0 \\
\hline Kentucky Blue Grass & 215 & 71.7 & 61 & 20.3 \\
\hline Meadow Fescue ......... & $\$ 9$ & 29.7 & 62 & 20.6 \\
\hline Orchard Grass ............ & 80 & 26.7 & 33 & 11.0 \\
\hline Hickory sp. & 21. & 7.0 & 45 & 15.0 \\
\hline 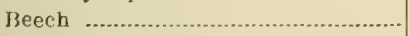 & $: 34$ & 11.3 & 43 & 14.3 \\
\hline White Oak ................... & 27 & 9.0 & 11 & 13.7 \\
\hline 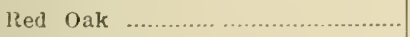 & 11 & 3.7 & 35 & 11.7 \\
\hline Black Oak ................. & 14 & 4.7 & 31 & 10.3 \\
\hline Amerlcan Elm ........ & 41 & 13.7 & 28 & $9 . .3$ \\
\hline Dock sp. ..................... & 25 & 8.3 & 93 & 31.0 \\
\hline Tullp Trce ........... & 26 & 8.7 & 36 & 12.0 \\
\hline Sassafras ...................... & 20 & 6.7 & 57 & 19.0 \\
\hline Wlld Ilydrangea ....... & 54 & 18.0 & 26 & 8.7 \\
\hline 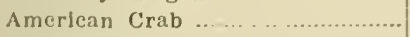 & 13 & 4.3 & 71 & 23.7 \\
\hline IIawthorn & $4 f$ & 15.3 & 12 & 30.7 \\
\hline Common Cinquefoll & 77 & 25.7 & 19.1 & 64.7 \\
\hline liack Raspberry .. & 184 & 61.3 & 37 & 12.3 \\
\hline 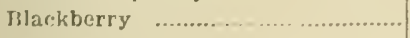 & 2 & 0.7 & 94 & $: 31.3$ \\
\hline 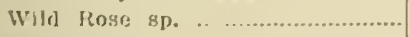 & 89 & 29.7 & 81. & 27.0 \\
\hline Red Clover ..... & $6 ; 2$ & 20.7 & 2!) & 9.7 \\
\hline
\end{tabular}




\begin{tabular}{|c|c|c|c|c|}
\hline \multirow[b]{2}{*}{ SPECIES } & \multicolumn{2}{|c|}{$\begin{array}{l}\text { WITHIN HORIZONTAL } \\
\text { PROJECTION OF CROWN }\end{array}$} & \multicolumn{2}{|c|}{$\begin{array}{l}\text { OUTSIDE HORIZONTAL } \\
\text { ProJeCTION OF CROWN }\end{array}$} \\
\hline & OCCURRENCES & $\begin{array}{c}\text { Per Cent } \\
\text { OCCURRence }\end{array}$ & OCCURRENCES & $\begin{array}{c}\text { Per Cent } \\
\text { OCCURRENCE }\end{array}$ \\
\hline White Clover .... & 131 & 43.7 & 48 & 16.0 \\
\hline Alsike Clover & 38 & 12.7 & 21 & 7.0 \\
\hline Sweet Clover & 42 & 14.0 & 20 & 6.7 \\
\hline Locust ............ & 32 & 10.7 & 41 & 13.7 \\
\hline 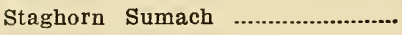 & 20 & 6.7 & 51 & 17.0 \\
\hline Dwarf Sumach & 46 & 15.3 & 58 & 19.3 \\
\hline Virginia Creeper .................. & 123 & 41.0 & 66 & 22.0 \\
\hline Wild Grape & 103 & 34.3 & 47 & 15.7 \\
\hline Violet sp. & 83 & 27.7 & 50 & 16.7 \\
\hline Mountain Laurel & 0 & 0.0 & 22 & 7.3 \\
\hline Blueberry & 0 & 0.0 & 31 & 10.3 \\
\hline Common Milkweed .......................... & 100 & 33.3 & 79 & 26.3 \\
\hline Common Speedwell & 46 & 15.3 & 82 & 27.3 \\
\hline Ironweed & 122 & 40.7 & 95 & 31.7 \\
\hline Goldenrod sp. & 133 & 44.3 & 107 & 35.7 \\
\hline Aster sp. & 162 & 54.0 & 79 & 26.3 \\
\hline
\end{tabular}

in some toxic substance produced by walnut roots. This study strongly supports the assumption that active antagonisms exist between black walnut and many other plants.

\section{Review of Literature}

Perry (12) investigated and made careful measurements on white pine (Pinus strobus L.) growing in association with black walnut and other tree species. Of a planting of sixty white pines made within sixteen feet of standing black walnut trees, many died ( 30 per cent of those within six feet of walnut trunks). Others were stunted and of poor form.

Smith (15) has pointed out the scarcity or absence of broomsedge and poverty grass under walnut trees. Schneiderhan (14) wrote extensively of injuries done apple trees in association with black walnut. On the other hand, Mattoon (10) failed to find evidence of such injury. Cook (4), Massey (9), and MacDaniels and Muenscher (8) made observations or conducted experiments on the effects of black walnut on potatoes and tomatoes. Massey also observed injury to alfalfa caused by standing black walnuts.

Pirone (13) has investigated injuries done by black walnuts to rhododendron and other Ericaceous plants. Jones and Morse (6) found apparent antagonism between walnut and shrubby cinquefoil (Potentilla fructicosa L.). 


\section{Black Walnut and Other Forest Trees}

Black walnut is an important timber tree in much of the Central Hardwoods region. It is one of the dominant species in the forest type known as "cove hardwoods," found commonly in the central and southern Appalachian region. Although walnut trees are scattered, and often scarce in the middle of dense timber stands, they are common in more open stands, at forest margins, and in natural or artificial openings.

It is important that the forester or timberland owner be aware of antagonisms that may exist between walnut and other timber species. This knowledge is of value in selecting the composition of mixed-tree plantings that will include walnut.

Table 1 shows that sixty species of trees, in varying numbers, were found growing within the root spread of mature black walnuts. This list includes most of the important timber species of the region investigated that would be likely to occur in association with walnut. A majority of the walnut trees investigated were growing in pastures or open fields. This may have accounted for the relatively small number of occurrences of many species.

The writer found no evidence of antagonism between walnut and any important timber species likely to occur naturally with it. In mixed-forest plantings, however, the situation may be different.

On a farm at French Creek, Upshur County, West Virginia, an experimental planting of red pine (Pinus resinosa Ait.) was made on an open hillside plot of about two acres. This plot contained three mature black walnut trees. Pines were planted six by six feet to within a short distance of the black walnut trunks.

Over the plot as a whole, the survival of red pines at the end of three years was slightly better than 80 per cent. Within the root spread of the black walnuts, however, all but two of the pines were dead within three years. These two were greatly stunted compared with those growing outside the walnut root spread. It was found that the roots of some of the dead pines were in contact with walnut roots.

This indicates that persons making mixed plantings of black walnut and other species should avoid the use of red pines and perhaps other pines. In eastern West Virginia, however, red cedar (Inniperus virginiana L.) grows freely in association with black walnut.

\section{Black Walnut, Shrubs, and Vines}

Table 2 shows that black walnut secms to offer a particularly favorable habitat for the growth of rertain slurubs and vincs. Even in 
heavily pastured areas there are usually seedlings of black raspberry, poison ivy (Rhus toxicodendron L.), Virginia creeper, and wild grape (Vitis spp.).

One of the most characteristic ground covers under black walnuts in this territory, both in open fields and in woodland borders, is a dense stand of black raspberry. This stand frequently grows up to the trunk of the tree. Table 2 shows black raspberry was found in varying quantities under 61.3 per cent of the trees examined.

The complete absence of shrubs of the Heath family (Ericaceae), as well as the virtual absence of blackberry (Rubus spp.), will be discussed later.

Since many of the shrubs and vines that regularly occur under walnut produce nuts and fruits of food value to game birds, songbirds, and mammals, the importance of black walnut to wildlife is emphasized.

\section{Black Walnut and Grasses}

The presence of grasses, often species of superior forage value, in considerable quantities under black walnut is commonly observed. Kentucky bluegrass occurred in varying quantities under 215 trees, or 71.7 per cent of the 300 trees studied. This grass was found under every walnut tree growing in open pastures, under most of those in woodland borclers, and under some in cultivated fields.

Other tame grasses that occurred commonly were timothy, 23 per cent; redtop, 29.3 per cent; tall oat grass, 18 per cent; meadow fescue, 29.7 per cent; and orchard grass, 26.7 per cent. Of wild grasses with inferior forage value, nimblewill was the most common. It occurred under 31 per cent of the walnut trees. Other fairly common wild grasses were broadleaved panic grass, 12.7 per cent; velvet grass (Holcus lanatus L.), 20 per cent; and brome grasses (Bromus spp.) 17 per cent.

The scarcity of two of the commonest "poor land" grasses in the region, broomsedge and poverty grass, was notable. Poverty grass was found under only 8 per cent of the trees, broomsedge under 4.3 per cent, although these grasses were often the most common outside the spread of the walnut trees. Even where these two grasses occurred under walnut, they were in sparse stands. Smith (15) has pointed out that broomsedge seldom grows within the shade of any tree, but that poverty grass is commonly found under maples, beeches, oaks, and hickories.

It appears that the black walnut trees create habitats that are favorable to cultivated grasses either on superior or poor sites. 


\section{Black Walnut and Grain Crops}

In the course of these studies, black walnut trees were observed in fields that had been planted to corn, oats, wheat, rye, and buckwheat. In no case was there sign of any inhibition or stunting of these crops greater than would be expected from shade and competition for water. Outside the shade zone, but in areas penetrated by walnut roots, these grain crops grew to normal size and productiveness.

\section{Black Walnut, Other Herbaceous Plants}

Under walnut trees where the forest cover is not too heavy, characteristic plant communities develop. These communities differ in many particulars from those found under other tree species. Many of the same herbaceous species are found both in open fields and woodlands.

Thirteen species and varieties of ferns, and two other pteridophytes, common horsetail (Equisetum arvense L.) and ground pine (Lycopodium complanatum var. flabelliforme Fernald), were found oftener than ten times under the three hundred trees studied. Of these, Christmas fern and ebony spleenwort (Asplenium platyneuron [L.] Oakes.) were common, the former occurring under 30.3 per cent, and the latter under 24.3 per cent of the walnuts. These persist even under heavy pasturing. Both the dissected and the common grape ferns also were common.

Clovers and other legumes of many species also are characteristic. White clover (Trifolium repens L.) is a common companion of Kentucky bluegrass, having been found under 43.7 per cent of the trees. Red clover ( $T$. pratense L.) and alsike clover ( $T$. hybridum L.) are somewhat less abundant.

Wood sorrel (Oxalis spp.), clammy cuphea (Cuphea petiola [L.] Kochne), wild carrot (Daucus carota L.), and common milkweed (Asclepias syriaca L.) also are abundant. Members of the mint family (Labiate) ale particularly well represented, with heal-all (Prunella inelgaris L.) being most frequently found.

The Composite fanily is extensively represented with ironweed (Vernonia spp.), goldenrod (Solidago spp.), aster (Aster spp.), daisy fleabane (Erigeron anmuus L. Pers.), ragweed (Ambrosia spp.), beggarticks (Bidens spp.), yarrow (Aclillea millefolium L.), ox-eye daisy (Chrysanthemum leucanthemum L.), golden ragwort (Senecio aureus L.), thistle (Cirsizm spp.), and dandelion (Taraxacum spp.). All of them were found under more than 100 of the 300 trees. 
Violets (Viola spp.), avens (Geum spp.), bedstraw (Galium spp.), and Indian tobacco (Lobelia inflata L.) are other common weeds found under walnut trees.

Although species may vary, this association of herbaceous plants remains fairly constant throughout the area, and also under walnuts examined in Michigan. Apparently, black walnut creates soil conditions that favor these plants.

\section{Black Walnut and Apple}

In Upshur County, West Virginia, observation was made on an apparently antagonistic relationship between black walnut and apple trees. In 1921, a three-acre plot was planted to apple trees. . Near the middle of the plot was a mature black walnut tree that bore nuts of excellent quality. It was left standing. Young apple trees were planted to within twenty feet of its trunk. On the remainder of the plot the survival of apple trees was about 90 per cent, but within three years all apple trees planted within the root spread of the walnut were dead. This was true even of those trees that could have been affected little, if any, by shade. Furthermore, there was complete survival of trees that received some shade from a sugar maple tree at one margin of the plot.

Apple trees were replanted to replace those that died, and these also were dead at the end of another three-year period. In the first planting, four of the apple trees were Grimes and two were Fall Rambo. All six trees replanted were Grimes.

In this investigation, apparent walnut injury to apple was observed in 21 instances; 2 in Upshur County, 1 in Barbour, 5 in Hampshire, 6 in Jefferson, 4 in Berkeley, 2 in Mineral, and 1 in Mason County, all in West Virginia. In each instance, young apple trees were planted within the root spread of a mature black walnut. When contact was made between apple and walnut roots, the apple trees showed serious injury. In most cases they died.

On seven occasions, mostly in the Shenandoah Valley, healthy apple trees were found growing quite close to standing black walnuts. In each case, however, examination showed the presence of a stone dike at or near the surface. This effectively separated apple and walnut roots. There was no apparent injury to apple from walnut leaf and hull residues. In no case, however, was a healthy apple tree found growing where its roots had made contact with walnut roots.

Most of the cases of apparent injury to apple were observed where walnuts were growing in fencerows. In commercial orchard sections 
it seems to be commonly accepted that apple and walnut will not grow together.

Supporting the observations made in this study are the findings of Schneiderhan (14), who reported in detail on eighteen instances of apparent walnut injury to apple trees. His observations were made in Frederick County, Virginia. For the area within the root spread of walnuts he used the term "toxic court" (as it affects apples). According to his figures, 16 walnut trees apparently caused the death of 48 apple trees, and serious injury to 14 others.

Schneiderhan found that trees of the Stayman variety were seemingly uninjured by contact with walnut roots. Mattoon (10) also tells of an instance where healthy Stayman trees were growing in close association with walnut. He doubts the validity of any walnut injury to apples.

Despite some conflicting evidence, however, it is apparent that in the region investigated, apple trees often are killed or seriously injured by mature black walnut trees. Persons planting young apple trees shouId avoid placing them within the root spread of black walnuts, unless the planting is made for experimental purposes.

\section{Black Walnut, Other Fruit Trees}

Field observation by the author fails to support the view, commonly held in commercial orchard regions, that contact with black walnut is injurious to fruit trees other than apples. In Berkeley County, West Virginia, four young peach trees were observed growing within the root spread of a large walnut. There were no rock dikes to prevent root contact, and the peaches were bearing heavily. The peach trees were apparently healthy. Peach trees were found growing under walnuts in 5 other situations, cherries were seen 3 times, plums 6 , pears 2, and quinces 1 . In no case was any injury or growth inhibition present.

\section{Black Walnut and Tomatoes and Potatoes}

In view of a widespread belief that tomatocs and potatoes do not thrive when grown under black walnut trees, special efforts were made to find these plants near walnuts. Twenty-six such cases were noted. In most instances tomatoes and potatoes growing within the root spread of walnuts were clead by July. In all cases the plants were scriously injured.

In Marshall County, West Virginia, a new garden had been made. A large walnut tree stood in a fencerow at one side of the garden. Rows 
of both tomatoes and potatoes ended within the shade of the tree. Both these vegetables growing within the walnut root spread were dead or dying on July 2, although plants outside the root spread were healthy. The owner said all of the plants received the same treatment. The walnut tree was high-crowned. Although shade may have been a factor, both lima and green beans were growing successfully within the root spread.

Several gardeners stated that they tried potatoes and tomatoes near black walnuts, but that they were not successful with them. They devoted such areas to other vegetables, growing them successfully despite the light walnut shade.

A Lewis County, West Virginia, garden had at one side a black walnut and a hickory of approximately the same size. Tomatoes growing under the walnut were wilted or dead in late June; those growing under the hickory showed some stunting where shade was more dense, but they were alive and unwilted. The shade of the hickory was much heavier than that of the walnut.

Cook (4) records a number of observations on walnut injury to tomatoes and potatoes. Massey (9) summarizes many such observations made by himself, and by A. W. Drinkard and F. D. Fromme. Of an attempt to study experimentally the effect of walnut on tomatoes, Massey writes:

In the center of a general garden, four rows of tomatoes were planted in the vicinity of a walnut tree . . The tomatoes were set out on May 20. In the latter part of June, plants began to wilt and die. The wilting of the plants was uneven over the area, and often one or two branches on the side of the plant became permanently wilted while the rest of the plant was healthy. Upon carefully removing the soil to expose the situation of the roots, it was found that in every case there was close contact between the tomato roots and those of the walnut. The plants wilted in a line sometimes diagonally across the patch, and sometimes parallel with the rows of tomatoes. This, it was found, was governed by the position of the walnut roots ... The direction of the (walnut) roots underground could be traced without removing any dirt, by observing the development of wilt in the tomato plants. The probable distribution of the roots was predicted and later verified by removal of the soil.

On the right of the tomato patch ... Irish potatoes were growing. There was a marked effect of the walnut on these potato plants, although it was not so distinct as on the tomatoes. Within the walnut-root invaded area of the garden were also growing, in good condition, beets, snap beans, and corn, none of which showed any signs of wilting. 
Using pieces of walnut bark in soil and water under laboratory conditions, Massey was able to produce wilting in tomatoes. MacDaniels and Muenscher (8) grew tomatoes in pots with two-year-old walnut seedlings. They were unable to detect any wilting or other deleterious effects to the tomatoes. Since young walnut seedlings put down a tap root before the lateral root system develops extensively, it is possible that no actual root contact was made. As Massey and others have emphasized, actual contact with walnut roots scems to be essential before injury to tomatoes and potatoes becomes apparent.

\section{Black Walnut and Alfalfa}

During the course of this investigation, eight situations were surveyed in which walnut could have influenced the growth of alfalfa. In most of these, a walnut was growing in a fencerow adjoining an alfalfa field. In each of these cases the alfalfa stopped at a line corresponding to the root spread of the walnut.

In one instance, west of Harpers Ferry, West Virginia, a black walnut tree of seventeen inches diameter was standing in the middle of an alfalfa field. The field was well-fertilized. Since the walnut was high-crowned, alfalfa was sown to within a foot or so of the trunk.

Within the root spread of the waInut, careful search failed to show a single alfalfa plant, although the outside stand was excellent. The line between the affected and unaffected areas was clear-cut. Under the walnut a stand of white, red, and alsike clover was developing. These plants had probably been present before the field was sowed to alfalfa.

In this and other cases, evidence of antagonism seemed definite, but the general picture was complicated by another observation. North of Point Pleasant, West Virginia, a mature walnut was found standing in the middle of an alfalfa field. Here also the alfalfa had been seeded up to the trunk of the high-crowned tree. The alfalfa, however, was growing vigorously within the root spread of the tree, and no differences in density of stand five feet from the trunk and one hundred feet away from it could be detected.

In support of the evidence that walnut frequently exerts a toxic effect on alfalfa, Massey (9) tclls of an alfalfa field on the Virginia Polytechnic Institnte Farm in which two large walnut trees were growing. Under these trees, alfalfa was completely replaced by grass, although it hacl been seeded up to the trunks. Under one of the trees the alfalfa-free area extended sixty-four feet from the trunk at one point. $A$ wahnut root was found at this distance from the tree. Allalfa had disappeared where it condel have made contact with walnut roots. 


\section{Black Walnut and Blackberries}

As mentioned earlier, a characteristic site for walnuts in the Appalachian region is on the slopes of dry and rather poor hillside pastures. In such pastures the three most common plants are broomsedge, poverty grass, and blackberries. Blackberries were found under only two of the three hundred trees examined. In these cases the plants were scattered and sparse, with no semblance of the dense thickets so common just outside the spread of the walnuts.

It seems unlikely that this can be a shade or moisture reaction. Blackberries grow freely under denser shade of many other trees. They also thrive in dry sites. A more plausible explanation might be found in the higher $\mathrm{pH}$ that prevails under walnuts. This is not entirely satisfactory, however. The $\mathrm{pH}$ under many of the walnuts examined, while uniformly higher than in surrounding areas, was no higher than in sites occupied by blackberries in other situations.

There remains the possibility of an antagonism induced by some toxic substance given off by walnut roots. Whatever it is, whether chemical or physical, it does not affect black raspberries, which are in the same genus as the blackberry. Blackberries often reach the root spread of the walnuts, stop there, and are replaced by black raspberries.

\section{Black Walnut and Ericaceous Plants}

Plants of the family Ericaceae are abundant and characteristic in acid soils throughout the Appalachian region. They occur in all sorts of sites-azaleas and blueberries in open fields, mountain laurel in brushy fields and thin woodlands, rhododendrons in dense shade, cranberries and other species in bogs.

It seems highly significant that no members of this extensive family were recorded where their roots could make contact with walnut. In a few isolated instances where Ericaceous plants, mainly mountain laurel (Kalmia latifolia L.), huckleberries (Gaylussacia spp.), and blueberries (Vaccinium spp.), were found under walnuts, rock ledges or dikes prevented any contact between walnut and heath roots.

Detailed observations on black walnut injury to Ericaceous plants are given by Pirone (13). Nursery-grown nine-year-old plants of Catawba rhododendren (Rhododendron catawbiense Michx.) were transplanted into a field containing walnut trees. Those that made contact with walnut roots (about five hundred plants) wilted and died by late August of the season in which they were transplanted. Roots not in contact with walnut were in a healthy and thriving condition. In one instance 
a rhododendron plant grew satisfactorily within two feet of a standing black walnut, but it was found that no contacts between root systems had been made.

The absence of plants of the Heath family from areas of walnut root spread has been explained by the higher $\mathrm{pH}$ under walnuts. This explanation is not wholly satisfactory. Although $\mathrm{pH}$ under walnuts is generally higher than in surrounding areas, many soils under walnuts are sufficiently acid to be within the tolerance ranges of the heaths. Furthermore, Pirone's observations, as well as the author's, show that members of this family do occur under walnuts where there is no root contact between the two plants.

This antagonism may be of considerable importance to nurserymen. Also, since black walnut is a favorite tree for lawn plantings, homeowners should recognize that rhododendrons, mountain laurel, azaleas, and other heaths are endangered if they are planted too close to walnuts.

\section{Other Walnut Species and Possible Antagonisms}

The only other native walnut within the range of black walnut is the butternut $(J$. cinerea L.). While this study was concerned primarily with black walnut relationships, scattered notes were taken on butternut trees at various places.

Butternut seems to have the faculty of encouraging Kentucky bluegrass and other domestic grasses as does black walnut. It is not nearly so abundant as black walnut in the region which this study covers, nor does it grow as large. Consequently, its significance in pastures, orchards, and gardens is less.

Massey and others have found that butternut has the same toxic ('ffect on tomatoes, potatoes, and alfalfa as cloes black wahnut. In the present investigation one case was lound, in Jefferson County, where young apple trees had clied after being planted in close association with butternut.

One of the earliest recorded instances of wahnut antagonism is that of Jones and Morse (6) relating to butternut in Vermont. They hat their atcention called to the lact that a common pasture platut, shrubby cincuefoil, was absent within the root speread of white walnut tuces. Of lheir own observations and those of $\Lambda$. H. Gilbert, they write:

Moreover, with such buttermuts, the 'dead line' for the weeds is pushed outward year by year as the tree cularges, so that the trec may be surrounded by a circle of dead and dying cincuetoil plants bordering the clean grassy plot under the tree. This antagonism is, we believe, attrilsutable rather to the root relations of the two plants, 
than to those of shade. Thus young butternuts from 2 to 8 feet high were observed to be surrounded by a circle which might be twice the diameter of the top of the tree, within which the weeds were dead, with dying plants bordering its margins. Such butternuts do not cause much shade. Moreover, young birch, beech, maple, cherry, apple, and pine trees in the same field showed no such striking relation to the death of the cinquefoil, healthy plants of the weed frequently growing close under their branches.

\section{Nature of Toxic Agent in Black Walnut}

Based on these and other studies, observations and experiments, there seems to be little doubt that black walnut produces a substance, or substances, that is often toxic to certain other plants. In this study, no attempt was made to investigate the nature of this toxic agent. There is, however, considerable evidence that bears on this problem.

Wheeler and Scott (16) reported studies on juglone, a substance derived from black walnut trees. Chemically, juglone is a napthaquinone. It is valuable in treating certain skin diseases. Some of the quinones are highly-efficient bacteriocides. One of them (benzoquinone) has been found very toxic to wheat seedlings.

As a result of his studies, Massey concludes (in part):

Roots of the affected plants were always in close contact with walnut roots; the toxic substance is not generally distributed in the soil around the walnut trees, but is localized in the vicinity of the walnut roots.

Walnut root bark contains a substance which is toxic to the roots of tomato plants grown in water culture.

It is likely that juglone, or some similar substance, is the toxic constituent of the walnut.

Davis (5) attempted to identify the toxic substance in black walnut trees with synthetic juglone. He reports as follows:

Attention has been called, by workers at the Virginia Agricultural Experiment Station, to the toxic effect of mature black walnut trees toward certain plant associations grown near them. Severe cases of similar injuries in apple orchards in Virginia have resulted in the death of apple trees which have been described. Following these observations, the present author, at the Virginia Agricultural Experiment Station, has sought to identify the toxic principle found in various parts of the walnut tree with juglone, which is known to occur throughout all parts of the plant. A substance has been extracted and purified, by a method for extracting juglone, from the hulls and roots of Juglans nigra. The purified and crystalline extract, in each case, has proved to be an exceedingly powerful toxin when injected into the stems of tomato and alfalfa 
plants. Juglone, or the 5-hydroxy-alpha-naphtha-quinone, has been synthesized by oxidation of alpha-naphtha-quinone. A comparison of its melting point with that of the toxic extract from Juglans nigra has shown these substances to be identical. Similar toxicity has been exhibited by both the synthetic and natural products.

Some investigators have attempted to explain the death of certain other plants in association with walnut through light factors, competition for moisture, and soil-fertility factors.

Concerning the first of these, Smith (15) points out, "Black walnuts on the average cast less shade than any other common tree of the area (southeastern Ohio)." As this study has shown, a number of plants that are inhibited by black walnut thrive under considerably denser shade than the walnuts supply. This would be particularly true with some members of the Heath family.

Competition for water cannot be accepted as a full explanation, since moisture was abundant in many cases of death of plants under walnut.

Concerning soil fertility, particularly the reactions of plants to the presence or absence of trace elements, it is impossible to be so sure of effects. It seems reasonable to suppose that plants in good sites and under favorable conditions of soil fertility will show increased tolerance to certain diseases or toxic substances. A combination of favorable circumstances may explain the thriving of alfalfa and other plants at certain times under black walnut. Conversely, when these plants die in association with walnut, it could be due to some fundamental lack in the soil.

It remains true, however, that in many situations and on many occasions, apple trees, tomatoes, potatoes, alfalfa, heaths, blackberries, and other plants have died when they came into contact with walnut roots. Furthermore, juglone has been shown to be highly toxic to tomatoes and alfalfa. The production of a toxic substance by walnut roots can explain all plants killings under walnut that have been observed. No other explanation yet put forth will do so.

\section{WALNUT AND THE SOIL}

RLACK walnut affects the soils in which it grows. The tree and Bits products create a soil situation highly favorable to Kentucky b) luegrass and other desirable grasses, encouraging to white clover and certain nther legumes, but which inhibits the growth of many plant species. 
Under the three hundred trees studied, $\mathrm{pH}$ readings were taken, with accompanying readings from outside the root spread of each tree. These readings were made with a pocket LaMotte Soil Test Kit. The readings are not highly accurate. They do give a good indication of soil $\mathrm{pH}$ in the samples tested. The results are given in Table 5.

Table 5. Effect of Black Walnut on pH of Soll (BASEd ON 300 Paired Tests)

\begin{tabular}{|c|c|c|c|}
\hline \multirow[b]{2}{*}{ SoIL } & \multicolumn{2}{|c|}{ AVERAGE PH OF SOIL SAMPLES } & \multirow[b]{2}{*}{ DIFFERENCE } \\
\hline & $\begin{array}{c}\text { Within } \\
\text { Toxic Court }\end{array}$ & $\begin{array}{l}\text { WITHOUT } \\
\text { TOXIC COURT }\end{array}$ & \\
\hline $\begin{array}{l}\text { Limestone and Calcareous } \\
\text { Shales above } \mathrm{pH} 6.0\end{array}$ & \multirow[t]{2}{*}{$6.4^{*}$} & 6.1 & \multirow[t]{2}{*}{0.3} \\
\hline $\begin{array}{l}\text { Sandstones and Acid Shales } \\
\text { below pH } 6.0\end{array}$ & & 5.1 & \\
\hline
\end{tabular}

*444 samples taken under 111 trees.

**756 samples taken under 189 trees.

In every case where soils outside the walnut root spread were highly acid, the $\mathrm{pH}$ under walnuts was higher. In soils that are derived from limestone, or that had been heavily limed, the differences within and without the walnut root spread were smaller. Even here, however, $\mathrm{pH}$ under walnuts was usually enough higher to show color differences in the tests.

Furthermore, it became apparent that the more acid the surrounding soil, the greater the improvement worked by walnut. Thus, when soils had readings of $\mathrm{pH} 5$ outside the walnut spread, the readings under walnut were between $\mathrm{pH} 5.5$ and 6 . In very acid soils $(\mathrm{pH} 4.5$ or lower) the differences under walnut were even greater. This was most apparent on poor hillsides overgrown with poverty grass, where, under the walnuts, there were often fairly good stands of Kentucky bluegrass.

Whether juglone or any other substance given off by walnut roots affects the fertility or composition of the soil remains to be shown. Walnut trees, however, do improve surrounding soil to the benefit of bluegrass and other important plants.

\section{Black Walnut and Pasture Improvement}

Since pasture planting of black walnut trees is being recommended by many soil conservation agencies, it may be well to examine the benefits to be expected from such a practice. Some of these benefits have already been discussed. Where poverty grass and broomsedge can be replaced by Kentucky bluegrass, the advantage to the livestock raiser is evident.

It seems worth pointing out again that the poorer the pasture, the 
greater the improvement walnuts will bring. Although walnuts grow more slowly and with poorer form on poor sites, they will grow on steep Appalachian hillsides.

Walnut trees in pasture land provide shade useful to livestock, without forming crowns so dense as to interfere with forage plants. There is a widely-held belief that cattle avoid grazing under black walnut trees. As a part of the present study, observations were made on the utilization of forage under black walnuts. It was found that both cattle and sheep make extensive use of such forage throughout the grazing season. This is particularly true in summer, since grass under walnut tends to stay green longer.

The reason for this widespread belief may be that livestock makes early and heavy use of forage under walnut. There is the inevitable accumulation of manure. Around these droppings, coarse grass springs up. It often is distasteful to cattle. The landowner, observing his pasture in midsummer, sees considerable quantities of unused grass under walnuts. He often concludes that animals have been avoiding it.

In 1939, technicians of a West Virginia soil conservation district made studies on walnut and locust-pasture combinations. Quadrats within and without the root spread of walnuts and locusts were measured and vegetation counted. From this study the following conclusions were drawn:

Preliminary studies indicate the following trends when a comparison of pasture vegetation is made under trees and with that outside. Under shade

a-Greater number of plants

b-Greater number of Kentucky bluegrass plants

c-Greater density of vegetation

$\mathrm{d}$-Decrease in erosion

The application which can be made of these data and observations are, first, locust and black walnut appear desirable if grown on eroded areas and terraces as well as in pastures, either natural seedings or plantings; second, they are acclimated and adapted to our soils, and are good for holding the soil.

Neel (11), working at the Middle Tennessee Experinent Station, reports on at study of cattle weight-gains in arcas planted to black walnut, black low ust, alad an muplanted check plot between them. The pioss were of equal size. The two were planted in 1926. Neel writes:

The walnut trees were spaced 25 fect apant and the locust trees 30) leet apart to hasten the time when the pasture would be: effectively shaded. They were to be pruned as they developed so that the sun could shine at the base of every tree. The objective was to obtain for the shaded plots not only light shade over the 
entire area for parts of every day, but also some sunshine for all the grass.

By the spring of 1932 the trees were large enough to be braced, so that pasturing could be started. Yearling beef steers, weighing 500 pounds in the spring, were used. These steers were weighed on and off the respective lots, and a record was kept of days grazed, as well as the yearly gains in weight that each lot produced.

For the years 1934-39, Neels' results showed an average yearly gain per acre of 214 pounds on the check plot, 189 on the locust plot, and 260 on the walnut plot.

Smith (15) states:

For every region there should be a theoretical 'ideal pasture tree' that would provide a maximum net benefit to the pastures. The studies in southeastern Ohio indicate that such a tree for this region should possess approximately the following characteristics:

1. Its period of leaf activity should extend only from June 15 to September 15.

2. Its canopy should admit approximately 50 per cent of tinc bright open sunlight to the grass during its period of leaf activity.

3. Its leaves should be small, fragile, and very high in minerals and nitrogen.

4. Its root system should extend to a great depth and be highly developed, but should feed mainly below the surface 4 inches of soil.

5. It should be a nitrogen-fixer.

6. It should be capable of establishment on poor upland sites.

7. It should possess high commercial value.

With these seven requirements as a standard it is relatively easy to indicate the ways in which trees fall short in their characteristics. The black walnut approaches the ideal in more ways than any other common species...

From these observations and conclusions, it seems clear that many pastures in this section would benefit greatly from well-spaced black walnut plantings. 


\section{Literature Cited}

1. Ashcroft, J. M., European Canker of Black Walnut and Other Trees. W. Va. Agr. Exp. Sta. Bull. 261, 1934.

2. Baker, F. S., Black Walnut: Its Growth and Management. U.S. Dept. of Agr. Bull. 933, 1921.

3. Broadfoot, W. M. and Pierre, W. H., "Forest Soil Studies: I. Relation of Rate of Decomposition of Tree Leaves to Their Acid-Base Balance and Other Chemical Properties." Soil Science, Vol. 48 (1939), pp. 329-48.

4. Cook, Mel T., "Wilting Caused by Walnut Trees." Phytopathology, Vol. 11 (1921), p. 346 .

5. Davis, E. F., "The Toxic Principle of Juglans nigra as Identified with Synthetic Jugione and Its Toxic Effects on Tomato and Alfalfa Plants." Am. Jour. Bot., Vol. 15 (1928), p. 620.

6. Jones, L. R. and Morse, W. J., "The Shrubby Cinquefoil as a Weed." 16th Annual Report, Vt. Agr. Exp. Sta. 1902-03.

7. Kline, L. V., "Tree Crops for the Tennessee Valley." Am. Forests, Vol. 47 (1949), pp. 470-72.

8. MacDaniels, L. H. and Muenscher, W. C., "Black Walnut Toxicity." Paper Read at 31st Annual Meeting, Northern Nut Growers Assoc., Roanoke, Va. (1940), pp. 1-8.

9. Massey, A. B., "Antagonism of the Walnuts (Juglans nigra L. and J. Cinera L.) in Certain Plant Associations." Phytopath., Vol. 15 (1925), pp. 773-84.

10. Mattoon, H. G., "A Commercial Black Walnut Venture." Am. Forests, Vol. 48 (1942), pp. 172-74.

11. Neel, L. R., The Effect of Shade on Pasture. Tenn Agr. Exp. Sta. Circ. 65, 1939.

12. Perry, G. S., "Some Tree Antagonisms." Proc. Pa. Acad. of Sci. VI (1932), pp. 1-6.

13. Pirone, P. P., "The Detrimental Effect of Walnut to Rhododendrons and Other Ornamentals." Nursery Disease Notes 11: Dept. of Plant Path., N. J. Agr. Exp. Sta. (mimeographed) 1938.

14. Schneiderhan, F. J., "The Black Walnut (Juglans nigra L.) as a Cause of the Death of Apple Trees." Phytopath, Vol. 17 (1927), pp. 529-40.

15. Smith, R. M., "Some Effects of Black Locusts and Black Walnuts on Sontheastern Ohio Pastures." Soil Science, Vol. 53 (1942), p. 14.

16. Wheeler, A. S. and Scott, J. W., "The Halogenation of Juglone, a New Type of Naphthalene Dye." Jour. Am. Chem. Soc., Vol. 41 (1919), pp. 833-41. 
